\title{
Variations, requirements and problems in obtaining ethics committees approval for multi-centers study in Bangkok and the metropolitan area
}

\begin{abstract}
Objectives: To describe the experience of applying to multi-center research ethics committees (MRECs) and local research ethics committees (LRECs), providing information regarding variations, requirements and problems when submitting for MRECs and LRECs approval in Bangkok and the metropolitan region.
\end{abstract}

Design: A retrospective evaluation of the processes of applying for the LRECs and the MRECs.

Setting: Sixty public government-funded hospitals based in Bangkok and the Metropolitan area.

Results: Fifty-nine hospitals replied; thirty granted chairman's approval, twenty-four required our proposal to be considered by additional research ethic committee and five disapproved the proposal. Also, the data are presented under 3 themes: organizational responses, time-requirements-local changes in the processing of obtaining ethical approval, and financial issues.

Conclusion: The diversity in practice of LRECs and MRECs in Bangkok and the Metropolitan area confirmed that without sufficient funding, a generous amount of time and a knowledge of Thai language, a multi-center study based in Thailand must be avoided.

Keywords: ethics committees approval, multi-center study, Bangkok and the metropolitan, Thailand
Volume 3 Issue I - 2018

\author{
Foongchomcheay A,' Eitivipart AC, ${ }^{2}$ \\ Guobadia $E^{2}$ \\ 'Chulalongkorn University, Thailand \\ 2University of Sydney, Australia
}

Correspondence: Anchalee Foongchomcheay, Chulapat 2 Building, Faculty of Allied Health and Sciences, Chulalongkorn University I54 Rama I Road, Bangkok, 10330, Thailand, Tel +66(0)96842535, Email Anchalee.F@chula.ac.th; a.foongchomcheay@gmail.com

Received: January 31, 2018 | Published: February 09, 2018

\section{Introduction}

Medical and healthcare services are established on evidencebased practice and research that are ultimately unavoidable involving human subjects. The primary aim of healthcare research involving human subjects is to gain more understanding of the etiology and pathogenesis of disease as well as to better the diagnostic, prophylactic, and therapeutic procedures. To protect the well-being of the subject, The World Medical Association has developed the Declaration of Helsinki to set as ethical philosophies, providing guidance to healthcare researchers who wish to conduct research studies involving human subjects. Healthcare research must adopt the ethical standards that promote respect, protect health, and right to all human beings. ${ }^{1}$ Each country has their own legal, ethical, and regulatory requirements for research on human subjects that align with the international standard. ${ }^{3}$ In order to conduct research, the investigator must: adopt the scientific principle; identify a clearly formulated experimental protocol; submit for consideration, comment, guidance; and gain approval from an appointed ethical review committee.

In many countries, healthcare research has been assessed by local researchethicscommittees(LRECs) thatwereestablishedindependently and individually within each hospital. Each LREC independently has its own ethical forms, regulations and different views on principal aspects of the research, causing frustrations to the researchers in obtaining local approval. The frustrating, expensive, and tedious processes of obtaining local ethical approval is problematic for research studies that need to carry out their research activities over a wide range of geographical area, especially for the multicenter research study. ${ }^{2-5}$ The variation of ethical requirements of LRECs has been complained as conceivably constraining valuable research. In Thailand, just like other countries, when applying to a large number of LRECs, researchers are encountering the problems regarding the administrative and ethical issues. ${ }^{4-6}$ Focusing on the specific area of Bangkok and the metropolitan region, the most developed cities of Thailand that comprising the biggest number of healthcare organizations, research institutes, and educational sectors, there is a Multi-center Research Ethics Committees (MRECs) based in Bangkok, but not all LRECs accept an approval from the MRECs. The aim of this study was to describe the experience of applying to MRECs and various LRECs, providing the most recent information regarding variations, requirements and problems when submitting for MRECs and LRECs approval in Bangkok and the metropolitan region.

\section{Methods}

The Department of Physical Therapy, Faculty of Allied Health Science, Chulalongkorn University started a retrospective survey study to investigate the number of new traumatic spinal cord injury cases from the medical records in 2015 within the public hospitals based in the Bangkok and the metropolitan region to calculate the incidence rate. Ethical approval was not considered essential for this type of study, but the proposed research received ethical approval from the the Ethics Review Committee for Research Involving Human Subjects, from the Health Science Group of Chulalongkorn University. The study involved sixty public hospitals covering six regions based in central Thailand: Bangkok, Nakhon Pathom, Pathum Thani, Nonthaburi, Samut Prakan, and Samut Sakhon. In early October 2016, a letter requesting a permission to 
perform data collection were sent to the chairman (or secretary) of each hospital with an approved proposal with information summarizing the background, aims, and methods of the proposed research. Also, the data collection forms were attached to the letter to guarantee that the patients' information would be collected anonymously and confidentially and that the collected data could not be traced back to patients. If additional or the institutional-based ethical approval was requested apart from the one that achieved, then an application form and institutional guideline were solicited and proceeded to obtain an ethical approval. To ensure the response rate, a followup telephone call was performed. The details of acquiring each ethical approval and the processes of getting permission to collect the data were documented. All were unaware that the responses were audited. Subsequently, the summary of the ethical approval procedures was analyzed and synthesized before presenting as a descriptive data. The data were presented under 3 themes: organizational responses, time-requirements-local changes in the processing of obtaining ethical approval, and financial issues.

\section{Results}

For the proposed identical study, the requirements to gain ethica approval are clearly different depending on the local organizations. All sixty chairmen (or the secretary) were contracted and replies were received from fifty-nine organizations, only one hospital was unable to obtain a contract.

Unfortunately, due to the limited funding and time commitment of completing the proposed project, the process of getting approval that takes further than 120 days was relinquished. Abandoning all the processes of obtaining approval from LRECs and MRECs which their application take 90-180 days from submission to first being considered and a further minimum of 30 days from the date of consideration to approval. Therefore, the data relevant to the LRECs and MRECs approval presenting in this article were based on the application form and institutional guideline of the individuals' committees. In addition, the mismatch between the funding of the project and the unexpected ethical approval processing fee, resulted in failure of the proposed project.

\section{Organizational responses}

Thirty hospitals granted chairman's approval, of these 13 required additional formal documents from the dean and the university as well as some proposal modifications before the chairman's approval were granted. The modifications were demanded and none of them asked for the same changes. Twentyfour hospitals required our proposal to be considered by additional research ethic committee. Of these hospitals, fourteen have their own ethic committee (LRECs) which only accept their institutional application form, eight hospital only accept an approval from Medical Service Department of Bangkok (MRECs based in Bangkok) and the other 2 hospitals only accept an approval from provincial health doctor of their city (MRECs based in their own city). In total, new 16 ethical applications must be re-written and submitted for the individual's committees' considerations. The frequency of the committee meeting ranged from monthly to bi-monthly. diagnosis services to other hospitals, and another one rejected to provide a reason for disapprovements.

\section{Time-requirements-local changes in processing of obtaining ethical approval}

For hospitals that granted the chairman's approval, the interval between sending an application to 30 chairmen and being granted approval ranged from 60 to 90 days. For the hospitals that need additional LRECs and MRECs approval, as it stated in the institutional guideline of each committee, it would take a minimum of 120 days for the whole process, excluding the time to prepare the paperwork. According to the application form and institutional guideline, to obtain the LRECs and MRECs approval, some specific requirements must be followed. All committees would accept the proposal in English language, only if the Thai version of that proposal was attached. Five of the LRECs requests that one of their healthcare staffs must take place in the proposed project as a co-investigator. All hospitals required that one of their staffs (mostly medical record staffs) must accompany a researcher during the data collection period. These are the prerequisite that needs to put in the proposal before submitting for further consideration of the committees. Almost all of the committees regulate that the complete application must be physically handed into the research department of the individuals' institutes and the payment of the processing fee must be made period to the submission of the application. An exception of one has an online application submission system.

\section{Financial issues}

The money spent for applying for multi-center research was not anticipated. The cost of the investigators, secretarial management, postage, photocopying and telephone calls were reached over 9,000 Thai Bath (THB). From 59 contracted hospitals, 48 have a processing fee (including LRECs and MRECs) which cost from 300 to 5,000 THB. Some hospitals also charged an extra cost for the amount of cases that were recruited in the proposed study (50 THB per case). Some hospitals revealed these informal unexpected costs after the approval were granted. An informal unexpected cost of processing fee and uncontrollable cost of case recruitment fee together with the long duration of the committees' consideration resulted in failure of the proposed project.

\section{Discussion}

The purposefully selected hospitals in this study cover a wide geographic area including six cities based in the central of Thailand. Therefore, a sensible conclusion could be drawn from our experience. Obtaining approval for multi-center studies is timeconsuming, especially for the studies that try to conduct their research in Thailand where information regarding the LRECs and MRECs couldn't be found electronically in the English language. Evidently, the main language for communication with administrative staffs or persons in charge is Thai, preventing international candidates who wish to be the main investigator and run their research activity in Thailand. Undoubtedly, until now there is no report of a successful large multi-center study in the healthcare field from Thailand. One major problem found during the initial process of identifying the prospective hospitals was that the outdated and/or incomplete contact information on their website were out of date and incomplete. In addition, the primary form of communication and the way of exchanging physical information between the researcher and the hospital was mainly conducted via telephone and mail with 
very few hospitals using e-mail. These ineffective and redundant communication methods causing delay and excessive payment to the proposed project. Furthermore, the variation between chairmen considerations and ethics committees' judgments inconsistent. This may be due to the diversity of the local needs, different practices, regulations, and guidelines. ${ }^{1-5}$ For example, there is no clear statement to distinguish the necessity between studies in which approval is and is not necessary. ${ }^{1}$ The delay in obtaining committees' approval for the proposed project, obviously, depend on the workloads, administrative management system and an experience of the committee in dealing with an external research project. The workloads of the hospital's staffs and the national or regional events are the main reasons for the delay and absent of the committees' meeting. Therefore, the submission of any external research project would be numbered and put on the waiting list which may or may not account for the next committees' meeting. Some hospitals mention that the queuing may take longer than twelve months. Additionally, some hospitals may have not dealt with an external research proposal; thus, they are unable to provide an immediate response which all depend on the chairman's consideration. Moreover, some hospitals were unable to assign a supportive staff to help and accompany researcher for the data collection period. The nature of an assigned voluntary position is that one would not get paid for an extra work for assisting the researcher. Consequently, the willingness to sign-up for this supportive position was incredibly low, which resulted in rejection of the proposal.

This is the first time ever, in which the information regarding ethical committees' approval in Thailand was reported. A researcher who wishes to conduct a multi-centers research in Thailand should be prepared to encounter difficulty in dealing with variations, requirements and problems that would possibly, unexpectedly and uncontrollable occur during the process of obtaining approval. In order to obtain ethical approval for a multi-location study in the most efficient way, an establishment of the central ethics committee at the regional and national level would overcome various problems of multi-center research. Also, the development of national standard ethics committee form would solve the problem of inconsistencies in preparing different documents for different committees.

\section{Conclusion}

There is too much diversity in the practice of LRECs and MRECs in Bangkok and the Metropolitan area. Without sufficient funding to cover the unexpected cost of conducting research and a generous amount of time for obtaining ethical approval that could possibly last over a year together with a knowledge of Thai language, a multicenter study based in Thailand must be avoided.

\section{Acknowledgements}

None.

\section{Conflict of interest}

The author declares no conflict of interest.

\section{References}

I. Ahmed AH, Nicholson KG. Delays and diversity in the practice of local research ethics committees.J Med Ethics. 1996;22(5):263-266.

2. Busby A, Dolk H. Local research ethics committees' approval in a national population study.J R Coll Physicians Lond. 1998;32(2): I 42-I45.

3. World Medical Association Declaration of Helsinki: ethical principles for medical research involving human subjects. The Journal of the American College of Dentists. 2014;8I (3): I4.

4. Harries UJ, Fentem PH, Tuxworth W, et al. Local research ethics committees. Widely differing responses to a national survey protocol.J $R$ Coll Physicians Lond. 1994;28(2): 150-154.

5. Maskell NA, Jones EL, Davies RJO. Variations in experience in obtaining local ethical approval for participation in a multi $\square$ centre study. QJM. 2003;96(4):305-307.

6. Tully J, Ninis N, Booy R, et al. The new system of review by multicentre research ethics committees: prospective study. BM].2000;320(7243): I I79_ 1182.

7. While AE. Ethics committees: impediments to research or guardians of ethical standards? BMJ. 1995;3 I I (7006):66 I. 